\title{
VIRULÊNCIA E ASPECTOS BIOLÓGICOS DE ISARIA JAVANICA (FRIEDER \& BALLY) SAMSON \& HYWELL-JONES SOBRE COPTOTERMES GESTROI (WASMANN) (ISOPTERA: RHINOTERMITIDAE)
}

\author{
R.S. Lopes ${ }^{1}$, V.M. Svedese ${ }^{1}$, A.P.A.S. Portela ${ }^{1}$, A.C. Albuquerque ${ }^{2}$, E.A. Luna-Alves Lima ${ }^{1}$ \\ ${ }^{1}$ Universidade Federal de Pernambuco, Centro de Ciências Biológicas, Departamento de Micologia, Labo- \\ ratório de Citologia e Genética de Fungos Filamentosos, Av. Prof. Nelson Chaves, CEP 50670-420, Recife, \\ PE, Brasil. E-mail: rs.lopes@hotmail.com
}

\section{RESUMO}

\begin{abstract}
O cupim asiático Coptotermes gestroi é considerado praga em áreas urbanas, causando danos expressivos em casas, edifícios e árvores. Esta pesquisa avaliou a eficiência de Isaria javanica URM4993 e I. javanica URM4995 sobre operários de C. gestroi. Os insetos foram pulverizados nas concentrações de $10^{4}$ a $10^{8}$ conídios/mL e mantidos em incubadora a $25 \pm 1^{\circ} \mathrm{C}$ e $80 \pm 10 \%$ de umidade relativa. Determinou-se a Concentração Letal $\left(\mathrm{CL}_{50}\right)$ e o Tempo Letal $\left(\mathrm{TL}_{50}\right)$, bem como foi quantificada a produção de conídios sobre os insetos mortos. Os aspectos biológicos referentes à germinação e esporulação, antes e após a infecção em C. gestroi, foram avaliados. O fungo foi virulento ao cupim, causando infecção em todas as concentrações utilizadas, sendo que I. javanica URM4993 foi mais eficiente, por causar mortalidade de $100 \%$, após o $6^{\circ}$ dia de inoculação $\left(\mathrm{CL}_{50}\right.$

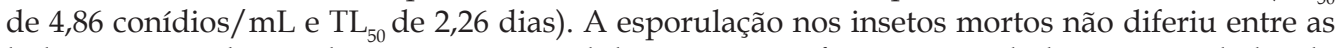
linhagens. Foi observado que o percentual de germinação foi maior nas linhagens reisoladas do que nas linhagens padrão. Contudo, I. javanica URM4993 apresentou maior potencial de esporulação. Esses resultados mostram o fungo como um candidato ao controle biológico desse cupim.
\end{abstract}

PALAVRAS-CHAVE: Controle biológico, Coptotermes gestroi, Isaria javanica, cupim.

\section{ABSTRACT}

VIRULENCE AND BIOLOGICAL ASPECTS OF ISARIA JAVANICA (FRIEDER \& BALLY) SAMSON \& HYWELL-JONES IN COPTOTERMES GESTROI (WASMANN) (ISOPTERA: RHINOTERMITIDAE). The Asiatic termite Coptotermes gestroi is considered a pestin urbanareas causing extensive damage to houses, buildings and trees. The present study evaluated the effectiveness of Isaria javanica URM4993 and URM4995 on workers of C. gestroi. Insects were sprayed with concentrations of $10^{4}$ at $10^{8}$ conidia/mL and kept in an incubator at $25 \pm 1^{\circ} \mathrm{C}$ and $80 \pm 10 \%$ relative humidity. Results were used to determine the lethal concentration $\left(\mathrm{LC}_{50}\right)$ and lethal time $\left(\mathrm{LT}_{50}\right)$. Conidia production on dead insects was assessed. Biological aspects related to germination and sporulation, before and after infection with $C$. gestroi were also assessed. The fungus was virulent to termites, causing infection at all tested concentrations, with I. javanica URM4993 being the most virulent, causing $100 \%$ mortality after six days of inoculation $\left(\mathrm{LC}_{50}\right.$ of $4.86 \times 10$ conidia/mL and $\mathrm{LT}_{50}$ of 2.26 days). Sporulation on dead insects did not differ between strains. The percentage of germination was greater for reisolated strains than for standard strains. However, I. javanica URM4993 showed greater sporulation. These results show that the fungus is a candidate for the biological control of termites.

KEY WORDS: Biological control, Coptotermes gestroi, Isaria javanica, termite.

\section{INTRODUÇÃO}

O cupim subterrâneo Coptotermes gestroi (Wasmann) (Isoptera: Rhinotermitidae) foi introduzido no sudeste brasileiro em 1923. É uma praga de expressivos danos às estruturas e aos objetos à base de celulose em áreas urbanas. Apesar do cupinzeiro central se formar no subsolo, o inseto produz ninhos secundários em substratos como madeiras, frestas de construção, interiores de paredes e pisos, caixas, cabos elétricos e telefônicos, causando sérios danos econômicos nas casas e nos edifícios. Esse cupim também ataca árvores, produtos armazenados, tecidos e outros materiais

${ }^{2}$ Universidade Federal Rural de Pernambuco, Departamento de Biologia, Recife, PE, Brasil. 
celulolíticos (Constantino, 1999; Fontes; Milano 2002; ZORZENON et al., 2006).

O controle preventivo dos cupins subterrâneos é realizado com aplicação de inseticidas de ação residual, como fipronil, imidacloprid, cipermetrina, permetrina, deltametrina, cyfluthrina e clorpirifós. Contudo, este método erradica apenas os ninhos secundários, possibilitando novas infestações pelos insetos sadios, provenientes do ninho central. Essas intervenções são dispendiosas e causam sérios problemas, em relação à poluição ambiental, contaminação das pessoas e aparecimento de insetos resistentes (GALLO et al., 2002; ZORZENON et al., 2006).

O controle biológico por fungo é uma alternativa eficiente no manejo de insetos-praga (AlvES, 1998). Esses patógenos apresentam alto potencial de disseminação por meio do contato social entre os membros da colônia (GRACE, 1997). A organização social dos cupins favorece a disseminação dos conídios dentro da colônia, facilitando o seu controle pelo tratamento das áreas de alimentação (RATH, 2000). Alguns entomopatógenos dos gêneros Beauveria, Metarhizium e Isaria apresentam resultados promissores em bioensaios, visando o controle de cupins das espécies $C$. acinaciformis (Froggatt) (MILNER et al., 1998), C. lacteus (Froggatt) (Milner, 2003), C. formosanus Shiraki, (Sun et al., 2003; WANG; Powell, 2003), e Nasutitermes coxipoensis (Holmgren) (Isoptera: Termitidae) (AlbuQuerque et al., 2005).

Isaria Persoon está entre os gêneros de fungos entomopatogênicos mais usados em programas de controle biológico, sendo empregado em escala comercial na Europa e nas Américas do Norte e Sul (FARIA; MAgAlHães, 2001). I. javanica (= Paecilomyces javanicus) (Frieder \& Bally) Samson \& Hywell-Jones é testado no controle de diversos insetos-praga. Li-nhagens dessa espécie já foram patenteadas (WRIGHT et al., 2003) e usadas em controle biológico dos cupins subterrâneos C. formosanus e Reticulitermes flavipes (Kollar) (Isoptera: Rhinotermidiae). O grupo de SCORSETTI et al. (2008) registrou a primeira ocorrência desse fungo na Argentina infectando Trialeurodes vaporariorum Westwood (Hemiptera: Aleyrodidae). No Brasil, a primeira ocorrência de I. javanica sobre a lagarta Lonomia obliqua Walker (Lepidoptera:Saturniidae) foi relatada por SРеснт et al. (2009), que comprovaram a sua patogenicidade sobre esse inseto.

Devido aos diversos prejuízos econômicos causados pelo C. gestroi nas áreas urbanas, faz-se necessário o uso de técnicas alternativas no controle deste inseto. O controle biológico utilizando fungos entomopatogênicos tem se mostrado promissor no controle de inúmeras pragas causadas por diversos insetos. O objetivo do trabalho foi testar o potencial de I. javanica, como agente biocontrolador do cupim C. gestroi, na busca de soluções ecológica e economicamente viáveis.

\section{MATERIAL E MÉTODOS}

\section{Linhagens fúngicas}

As linhagens I. javanica URM4993 e I. javanica URM4995 foram cedidas da Coleção de Cultura de Fungos, da Micoteca/URM do Departamento de Micologia/UFPE.

\section{Ação de Isaria javanica sobre Coptotermes gestroi}

As linhagens I. javanica URM4993 e I. javanica URM4995 foram cultivadas em tubos de ensaio contendo sabouraud-dextrose-àgar (SAB) durante12 dias e foram produzidas em arroz cozido conforme a metodologia de VILAS BoAs et al. (1996). Em seguida, um grama de arroz colonizado pelo o fungo foi homogeneizado em Erlenmeyer com 100 mL de água destilada estéril e espalhante adesivo (Tween 80) a 0,5\%. A suspensão foi quantificada em câmara de Neübauer e a partir desta foram realizadas diluições sucessivas para a obtenção das concentrações $10^{8}$, $10^{7}, 10^{6}, 10^{5}$ e $10^{4}$ conídios $/ \mathrm{mL}$.

O cupim foi coletado de dois cupinzeiros localizados no Campus da UFPE. Fragmentos do ninho foram cortados, transferidos para potes plásticos e levados para o Laboratório de Controle Biológico/ UFPE, no qual o material foi analisado, sendo os operários selecionados para os bioensaios.

$\mathrm{O}$ bioensaio sobre virulência foi realizado em triplicata simultaneamente, com seis tratamentos constituídos de um grupo controle, contendo água destilada estéril e espalhante adesivo (Tween $80)$ a $0,5 \%$ e de cinco tratamentos nas concentrações $10^{4}, 10^{5}, 10^{6}, 10^{7}$ e $10^{8}$ conídios/mL. Para cada concentração foi utilizado cinco repetições e a unidade experimental foi constituída por 10 cupins. Os operários foram imobilizados por um minuto à temperatura de $-2^{\circ} \mathrm{C}$ e pulverizados com pulverizador manual De Vilbiss $\mathrm{n}^{\circ} 15$, com $0,5 \mathrm{~mL}$ de cada suspensão. Em seguida, os cupins foram transferidos para placas de Petri forradas com papel filtro umedecido e com um pedaço de madeira, para abrigo e alimentação. As placas foram mantidas em câmara climatizada B.O.D. (Biological Oxigen Demand) com temperatura de $25 \pm 1^{\circ} \mathrm{C}$ e umidade relativa de $80 \pm 10 \%$. Foram realizadas observações diárias até o $12^{\circ}$ dia após a pulverização, sendo os insetos mortos removidos e colocados em câmara úmida, para confirmação da mortalidade causada pelo entomopatógeno. Os dados obtidos foram empregados na determinação 
do percentual de mortalidade, Concentração Letal $\left(\mathrm{CL}_{50}\right)$ e Tempo Letal $\left(\mathrm{TL}_{50}\right)$.

Produção de conídios de Isaria javanica sobre Coptotermes gestroi

A esporulação sobre $C$. gestroi foi determinada utilizando os insetos mortos oriundos dos bioensaios. Quatro operários expostos à concentração $10^{8}$ conídios/mL foram imersos em $4 \mathrm{~mL}$ de solução Tween 80 a 0,05\%. Em seguida agitada por três minutos em Vortex e procedeu-se à quantificação dos conídios, em câmara de Neübauer. Foram realizados três bioensaios, cada um composto por cinco repetições.

\section{Análise dos aspectos biológicos de Isariajavanica}

Uma parcela dos operários colonizados pelas linhagens fúngicas foi desinfetada com hipoclorito de sódio a $4 \%$, álcool $70 \%$ e água destilada autoclavada por dois segundos, três minutos e três minutos, respectivamente, conforme Alves et al. (1998). Os operários foram transferidos para placas de Petri, com SAB acrescido de antibiótico Cloranfenifcol a $2 \%$ e incubados em B.O.D. $\left(25 \pm 1^{\circ} \mathrm{C}\right)$ até a esporulação do fungo. Em seguida, inóculos do patógeno foram transferidos para tubo de ensaio para posterior análise dos aspectos biológicos. A viabilidade das linhagens foi avaliada por meio do percentual de germinação dos conídios e da esporulação, em SAB, antes e após o reisolamento de C. gestroi. Considerou-se como fungo-padrão (P) I. javanica URM4993 e URM4995, da Micoteca/ URM, e o fungo-reisolado (R) as mesmas linhagens reisoladas dos cupins-operários. Para estimar o percentual de germinação utilizou-se a metodologia de Alves; Pereira (1998). A produção de conídios foi avaliada pela inoculação de $0,1 \mathrm{~mL}$ de uma suspensão de $10^{8}$ conídios/ $\mathrm{mL}$ de cada linhagem em placas de Petri contendo SAB, em quintuplicata, e espalhada com o auxílio de uma alça de Drigalsky sobre a superfície do meio de cultura. As placas foram mantidas em B.O.D $\left(25 \pm 1^{\circ} \mathrm{C}\right)$ e, após 3, 6, 9 e 12 dias de crescimento, foram adicionados às placas $5 \mathrm{~mL}$ de uma solução de etanol a $75 \%$, para matar e secar os conídios. Posteriormente, as placas foram lavadas dez vezes com $10 \mathrm{~mL}$ de solução Tween 80 a 0,05\%. O lavado foi coletado em frasco Erlenmeyer de $250 \mathrm{~mL}$, agitado por cinco minutos e quantificado em câmara de Neübauer.

O delineamento experimental empregado foi inteiramente casualizado, para todos os experimentos. A análise de Probit foi utilizada para obtenção dos valores de $\mathrm{CL}_{50} \mathrm{eTL}_{50}$, utilizandoo ProgramaPOLOPC Para a análise dos experimentos foi utilizado o teste $F$ (ANOVA)e, em seguida, as médias foram comparadas pelo teste de Duncan a $5 \%$ de probabilidade, ambos utilizando o Programa SAEG (versão 5.0).

\section{RESULTADOS E DISCUSSÃO}

\section{Ação de Isaria javanica sobre Coptotermes gestroi}

Os operários foram suscetíveis às linhagens de $I$. javanica, as quais apresentaram alta virulência nos diferentes tratamentos, diferindo estatisticamente do grupo controle, noqual não foi observada mortalidade causada pelo fungo (Tabela 1). I. javanica URM4993 foi significativamente mais virulenta a C. gestroi, atigindo no $6^{\circ}$ dia deinoculação índices demortalidade de 89,33 e $100 \%$, nos tratamentos com $10^{7}$ e $10^{8}$ conídios $/ \mathrm{mL}$, respectivamente. Opercentual demortalidade dogrupo controle foi discreto $(8,67 \%)$, sendo constatada diferença significativa entreocontroleeos tratamentoscom o fungo a partir da concentração $10^{5}$ conídios $/ \mathrm{mL}$. No $9^{\circ}$ dia deexposição I.javanica URM4993 causou 100\% de mortalidade na concentração $10^{7}$ conídios $/ \mathrm{mL}$, diferindo estatisticamente de I. javanica URM4995, que atingiu mortalidade de $87,33 \%$ com a mesma concentração. No $12^{\circ}$ dia pós-inoculação, 100\% dos insetos pulverizados com I. javanica URM4993 foram mortos a partir de $10^{6}$ conídios $/ \mathrm{mL}$, enquanto que para $I$. javanica URM4995 o mesmo foi evidenciado a partir $10^{7}$ conídios/mL.

Tabela 1 - Percentagem média de mortalidade de Coptotermes gestroi em diferentes dias após a infecção por Isaria javanica.

\begin{tabular}{|c|c|c|c|c|c|c|c|c|}
\hline \multirow{2}{*}{ Tratamento } & \multicolumn{2}{|c|}{3 dias } & \multicolumn{2}{|c|}{6 dias } & \multicolumn{2}{|c|}{9 dias } & \multicolumn{2}{|c|}{12 dias } \\
\hline & URM4993 & URM4995 & URM4993 & URM4995 & URM4993 & URM4995 & URM4993 & URM4995 \\
\hline Controle & $3,33 \mathrm{C}, \mathrm{a}$ & $3,33 \mathrm{D}, \mathrm{a}$ & $8,67 \mathrm{E}, \mathrm{a}$ & $8,67 \mathrm{D}, \mathrm{a}$ & $11,33 \mathrm{E}, \mathrm{a}$ & $11,33 \mathrm{~F}, \mathrm{a}$ & $15,33 \mathrm{D}, \mathrm{a}$ & $15,33 \mathrm{E}, \mathrm{a}$ \\
\hline $10^{4}$ & $6,67 \mathrm{C}, \mathrm{a}$ & $3,67 \mathrm{D}, \mathrm{a}$ & $21,33 \mathrm{E}, \mathrm{a}$ & $15,33 \mathrm{D}, \mathrm{a}$ & $37,33 \mathrm{D}, \mathrm{a}$ & $32,67 \mathrm{E}, \mathrm{a}$ & $54,67 \mathrm{C}, \mathrm{a}$ & $48,00 \mathrm{D}, \mathrm{b}$ \\
\hline $10^{5}$ & 14,67 C,a & $11,33 \mathrm{CD}, \mathrm{a}$ & $40,00 \mathrm{D}, \mathrm{a}$ & $33,33 \mathrm{C}, \mathrm{a}$ & $58,00 \mathrm{C}, \mathrm{a}$ & 50,67 B,a & $73,33 \mathrm{~B}, \mathrm{a}$ & $59,33 \mathrm{C}, \mathrm{b}$ \\
\hline $10^{6}$ & 33,33 B,a & 21,33 BC, $\mathrm{a}$ & $55,33 \mathrm{C}, \mathrm{a}$ & $53,33 \mathrm{~B}, \mathrm{a}$ & 89,33 B,a & $78,00 \mathrm{C}, \mathrm{b}$ & $100,00 \mathrm{~A}, \mathrm{a}$ & 93,33 B,b \\
\hline $10^{7}$ & $48,67 \mathrm{~A}, \mathrm{a}$ & $30,67 \mathrm{AB}, \mathrm{b}$ & 89,33 B,a & $66,67 \mathrm{AB}, \mathrm{b}$ & $100,00 \mathrm{~A}, \mathrm{a}$ & 87,33 B,b & $100,00 \mathrm{~A}, \mathrm{a}$ & $100,00 \mathrm{~A}, \mathrm{a}$ \\
\hline $10^{8}$ & $62,00 \mathrm{~A}, \mathrm{a}$ & $43,33 \mathrm{~A}, \mathrm{~b}$ & $100,00 \mathrm{~A}, \mathrm{a}$ & $78,00 \mathrm{~A}, \mathrm{~b}$ & $100,00 \mathrm{~A}, \mathrm{a}$ & $100,00 \mathrm{~A}, \mathrm{a}$ & $100,00 \mathrm{~A}, \mathrm{a}$ & $100,00 \mathrm{~A}, \mathrm{a}$ \\
\hline $\mathrm{CV}(\%)$ & \multicolumn{2}{|r|}{30} & \multicolumn{2}{|c|}{14,12} & \multicolumn{2}{|c|}{6,58} & \multicolumn{2}{|c|}{3,00} \\
\hline
\end{tabular}

Médias seguidas de mesmas letras, maiúscula na coluna e minúscula na linha, não diferem estatisticamente entre si, pelo teste de Duncan $(\mathrm{P}=0,05)$. 


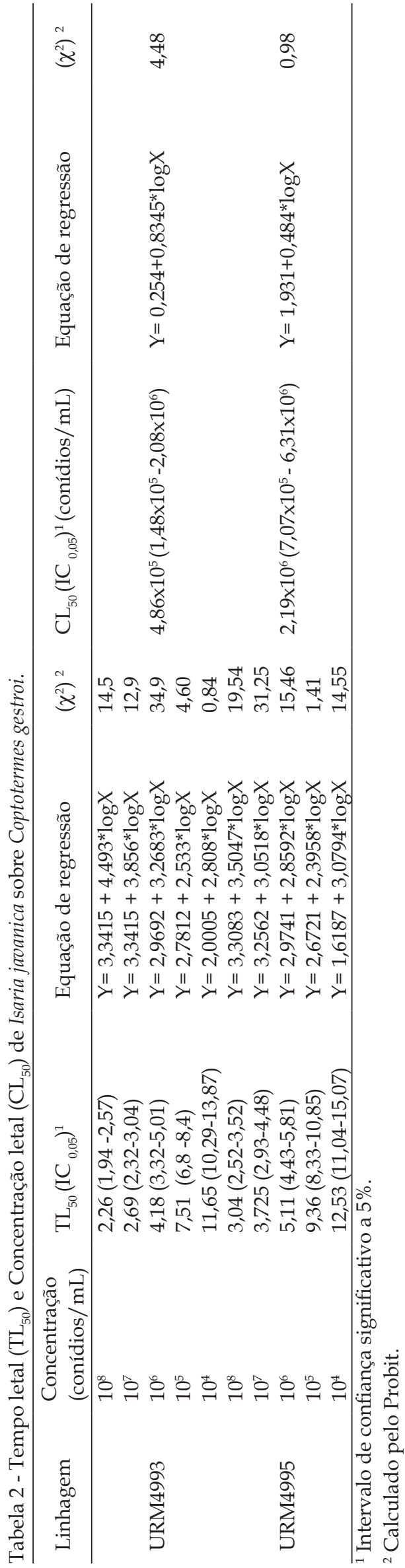

Espécies de Isaria são utilizadas com sucesso nos bioensaios em laboratório de cupins-praga. Resultados semelhantes à pesquisa foram encontrados por MeikLe et al. (2005) ao isolarem I. fumosorosea (= P. fumosoroseus) (Wize) Brown \& Smith de C. formosanus e, em seguida, testaram sobre operários. Os autores constataram que o fungo causou mortalidade de $100 \%$ na concentração $10^{7}$ conídios $/ \mathrm{mL}$, após dez dias, bem como uma alta esporulação sobre os cupins tratados, demonstrando que esse pode ser utilizado nos testes de biocontrole no campo. Ainda, YANAGAWA et al. (2008) verificaram que I. fumosorosea causou mortalidade de 40 e $100 \%$ de C. formosanus quando pulverizado nas concentrações de $10^{7} \mathrm{e}$ $10^{8}$ conídios $/ \mathrm{mL}$, respectivamente, após seis dias de avaliação. Por outro lado Wright et al. (2003) patentearam linhagens não-repelentes de I. javanica e I. fumosorosea virulentas aos cupins. Os conídios desses fungos são facilmente disseminados entre os indivíduos da colônia e causam rápida mortalidade nos cupins Reticulitermes flavipes Kollar (Isoptera: Rhinotermidae) e C. formosanus.

Testes com outros fungos foram feitos, inclusive com B. bassiana sobre C. formosanus e os resultados mostraram que $100 \%$ dos operários morreram após sete dias de exposição ao patógeno (WRight et al., 2002). Também a mortalidade de $100 \%$ dos operários de C. formosanus por M. anisopliae foi constatada após 14 dias, em bioensaios utilizando a concentração 2 x $10^{6}$ conídios $/ \mathrm{mL}$ (WRIGHT et al., 2005). Contudo, a mortalidade de $100 \%$ dos operários de C. gestroi foi inferior aos resultados obtidos por WRIGHT et al. (2005), mostrando assim, o potencial das linhagens de I. javanica no controle deste cupim. Os resultados expostos na Tabela 1 mostram a ação patogênica de I. javanica sobre C. gestroi, indicando maiores índices de mortalidade desse inseto com o aumento da concentração utilizada, superiores a $50 \%$ após $12^{\circ}$ dia de observação. Ainda, comparando a mortalidade dos operários, causadas pelas duas linhagens, I. javanica URM4993 foi mais virulenta, provocando a morte dos insetos em menor tempo, sugerindo maior eficiência no controle desse cupim.

Avaliação da Concentração Letal e Tempo Letal de Isaria javanica sobre operários de Coptotermes gestroi

Utilizando a análise de Probit foi determinada a $\mathrm{CL}_{50}$ e o $\mathrm{TL}_{50}$ das linhagens de I. javanica, considerando os resultados da mortalidade sobre os cupins nas diferentes concentrações utilizadas. I. javanica URM4993 apresentou $\mathrm{CL}_{50}$ de $4,86 \times 10^{5}$ conídios/ $\mathrm{mL}$, sendo este valor inferior ao apresentado por I. javanica URM4995, que demonstrou $\mathrm{CL}_{50}$ de 2,19 x $10^{6}$ conídios $/ \mathrm{mL}$ (Tabela 2). Embora utilizando organismos diferentes, HANEL (1981) observou resul- 
tados semelhantes, quando testou a eficiência de $M$. anisopliae contra N. exitiosus (Hill) nas concentrações de $1,29 \times 10^{7}$ e $1,29 \times 10^{3}$ conídios/ $\mathrm{mL}$, e verificou $\mathrm{CL}_{50}$ de $5,13 \times 10^{5}$ e $3,56 \times 10^{4}$ conídios/mL após oito e 11 dias, respectivamente. Todavia, MiLNER et al. (1997) analisaram a eficiência de M. anisopliae FI-610 contra operários de $N$. exitiosus e de C. acinaciformis e obtiveram $\mathrm{CL}_{50}$ de $7,9 \times 10^{6}$ e de $5,8 \times 10^{5}$ conídios/ $\mathrm{mL}$, para as espécies de cupins, respectivamente, obtendo resultados satisfatórios.

O valor do TL confirmou a eficiência de I. javanica sobre C. gestroi. Os valores de $\mathrm{TL}_{50}$ decresceram com o aumento da concentração de conídios nas soluções utilizadas. Desse modo, quanto mais elevada foi a concentração conidial empregada, maior foi a variação de mortalidade entre os tratamentos, justificando a importância da determinação do $\mathrm{TL}_{50}$ e do limite de confiança, com as variações mínimas e máximas para a análise da eficiência das linhagens testadas (Tabela 2). I. javanica URM4993 foi mais virulenta, fato evidenciado pelo menor tempo letal observado em todas as concentrações testadas. Os valores de $\mathrm{TL}_{50} \mathrm{se}$ correlacionaram positivamente com a $\mathrm{CL}_{50}$ e com os percentuais de mortalidade, sendo a concentração $10^{8}$ conídios/mL mais eficiente para as duas linhagens, com $\mathrm{TL}_{50}$ de 2,26 e 3,04 dias para I. javanica URM4993 e URM4995, respectivamente, mostrando, assim, a maior eficiência de I. javanica URM4993 no controle de C. gestroi. Loureiro; MonTEIRO (2004) obtiveram resultados simlares, quandoavaliaram a patogenicidade de I. farinosa ( $=P$. farinosus) (Holm: Fries) Fries contra operárias da formiga Atta sexdens sexdens Linnaeus (Hymenoptera: Formicidae, e determinaram $\mathrm{TL}_{50}$ de 2,64 dias, ao utilizarem $10^{8}$ conídios/mL.

A determinação da CL em experimentos com entomopatógenoséumimportante parâmetrobiológico quando aplicado adequadamente (Alvesetal., 1998), visto que o potencial do fungo consiste na estimativa

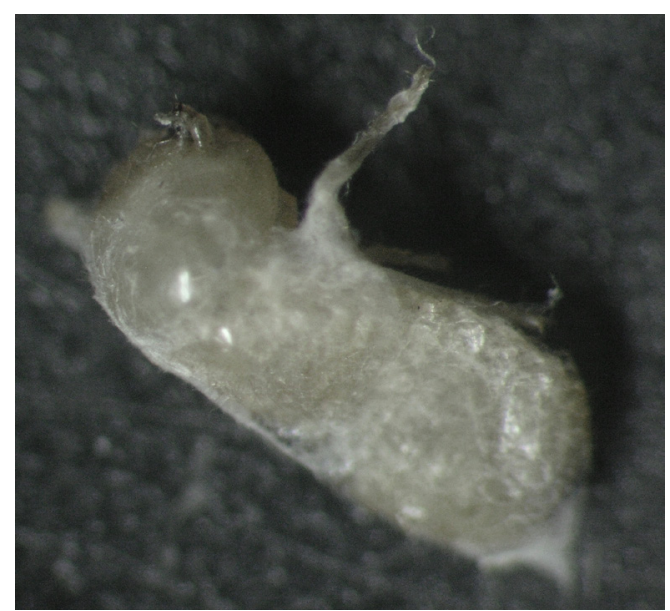

Fig.1 - Operário de Coptotermes gestroi colonizado (2 dia) por Isaria javanica URM4995. (50X). da $\mathrm{CL}_{50}$, sendo este parâmetro mais eficiente para testes de seletividade do patógeno ao inseto alvo. Segundo SiLva et al. (2003), o TL ${ }_{50}$ é utilizado como um parâmetro auxilar na determinação da virulência, onde é mais importante a redução populacional da praga do que a rapidez desse processo.

Produção de conídios de Isaria javanica sobre Coptotermes gestroi

A Tabela 3 apresenta a produção de conídios de I. javanica sobre cadáveres de operários de $C$. gestroi, como também mostra que não houve diferença significativa entre as linhagens utilizadas, de acordo com o teste de Duncan $(\mathrm{p}=0,05)$. A esporulação sobre o cupim apresentou médias de $1,48 \times 10^{7}$ e $1,08 \times 10^{7}$ conídios/mL, para I. javanica URM4993 e URM4995, sendo eficientes na produção de conídios sobre C. gestroi. A produção de conídios também foi analisada por AlbUQUERQUe et al. (2005) utilizando M. anisopliae var. anisopliae sobre operários e soldados de N. coxipoensis. Como resultado, constataram médias de $385 \times 10^{7}$ e 550 x $10^{7}$ conídios $/ \mathrm{mL}$. Também experimentos realizados sobre a esporulação de $B$. bassiana e M. anisopliae sobre C. formosanus foram feitos por Sun et al. (2002) que verificaram um aumento significativo da esporulação após $11^{\circ}$ dia da morte do inseto.

Tabela 3 - Produção de conídios de Isaria javanica sobre Coptotermes gestroi.

\begin{tabular}{lc}
\hline Linhagem & Esporulação $\left(10^{7}\right.$ conídos $\left./ \mathrm{mL}\right)$ \\
\hline URM4993 & $1,48 \mathrm{~A}$ \\
URM4995 & $1,08 \mathrm{~A}$ \\
\hline CV & $46,35 \%$ \\
\hline
\end{tabular}

Médias seguidas de mesma letra, na coluna, não diferem estatisticamente entre si, pelo teste de Duncan $(P=0,05)$.

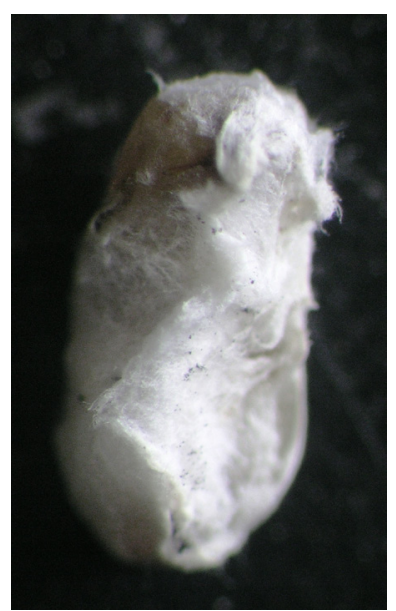

Fig. 2 - Operário de Coptotermes gestroi colonizado (7 dia) por Isaria javanica URM4993. (50X). 
A formação de micélio das linhagens de I.javanica sobre $C$. gestroi foi semelhante à descrita por Sun et al. (2002) e AlbuQUERQUe et al. (2005), iniciando em volta das peças bucais e antenas e, em seguida, nas membranas intersegmentais e ao redor das patas. A formação micelial ocorreu no $2^{\circ}$ dia após a morte dos insetos, com aspecto ralo e hialino (Fig. 1) e, após o $7^{\circ} \mathrm{dia}$, as linhagens colonizaram todo inseto, apresentando micélio de aspecto cotonoso e de cor branca (Fig. 2). Estas características são semelhantes às descritas por SAMSON (1974) e SAMSON et al. (1988) ao descreverem o desenvolvimento de espécies de Isaria sobre insetos-praga.

A esporulação é um fator importante na disseminação e permanência dos fungos entomopatogênicos nas populações de insetos, em especial nos cupins, devidoao contato social que permite a transferência do inóculo, de cupins infectados para os não-infectados; além de aumentar o potencial do inóculo no ambiente do hospedeiro, o que é essencial para que esses patógenos sejam bons biocontroladores de insetos-praga.

\section{Análise dos aspectos biológicos de Isariajavanica}

A germinação diferiu estatisticamente entre o fungo-padrão e o reisolado de cada linhagem, não sendo observada diferença significativa entre as linhagens, nas quais os percentuais de germinação variaram de 94,04 e $89,92 \%$ para o fungo-padrão e de 98,24 e $97,76 \%$ para o reisolado de I. javanica URM4993 e URM4995, respectivamente (Tabela 4). A germinação é um fator decisivo no controle microbiano, pois uma taxa elevada de esporos germinados é diretamente proporcional à virulência apresentada pelas linhagens fúngicas (ALVES, 1998). A esporulação de I. javanica URM4993 diferiu estatisticamente a partir do $6^{\circ}$ dia, sendo a esporulação maior para o reisolado, o que não ocorreu com $I$. javanica URM4995, que não apresentou diferença significativa na produção de conídios nos períodos analisados. Os dados estatísticos demonstraram que as linhagens se comportaram diferentemente, I. javanica URM4993 apresentou maior esporulação, com maior conidiogênese para reisolado $\left(21,64 \times 10^{7}\right.$ conídios/mL) após12 dias (Tabela 4). A produção de conídios viáveis em meio de cultivo e sobre o cadáver deinsetos consiste em um importante parâmetro para avaliar o potencial do patógeno e auxilia na seleção de linhagens mais virulentas a serem utilizadas em formulações e aplicação em programas de controle de pragas (AlmEIDA et al. 2005).

Das linhagens testadas, I . javanica URM4993 foi mais virulenta aos operários de C. gestroi, causando $100 \%$ de mortalidade após o $6^{\circ}$ dia de inoculação. A alta esporulação das linhagens sobre os insetos mortos confirma a competência deste fungo e sua permanência sobre o cupim. O fungo apresentou boa viabialidade antes e após o reisolamento, com ótima média de germinação de conídios e esporulção. Estes dadosindicamo potencial das linhagens para emprego no controle de C. gestroi, principalmente I. javanica URM4993, que se apresenta como uma alternativa viável como agente biocontrolador desta praga.

\section{AGRADECIMENTOS}

Os autores agradecem ao Pesquisador Luiz Roberto Fontes, da Superientendência de Controle de Edemias (Sucen), pela confirmação da espécie do cupim estudada. Ao Pesquisador Venésio Felipe dos Santos, do Instituto de Pesquisa Agropecuária (IPA) e ao Dr. Franscico Braga da Paz Júnior, do Instituto Federal Tecnológico de Pernambuco (IFET-PE), pelo auxílio nas análises estatísticas. Ao Conselho Nacional de Pesquisas (CNPq), pela concessão da Bolsa de Mestrado, ao primeiro autor e ao $\mathrm{CNPq}$ pelo apoio financeiro (Edital Universal).

Tabela 4 - Médias de germinação e produção de conídios de Isaria javanica antes e após a passagem por Coptotermes gestroi.

\begin{tabular}{|c|c|c|c|c|c|}
\hline \multirow{2}{*}{ Tempo(horas/dias) } & \multirow{2}{*}{ Aspectos Biológicos } & \multicolumn{4}{|c|}{ Linhagens } \\
\hline & & & URM4993 & & URM49995 \\
\hline \multirow[t]{3}{*}{16 horas } & Germinação (\%) & $\mathrm{P}^{1}$ & $94,08 \mathrm{~B}, \mathrm{a}$ & $\mathrm{R}^{2}$ & $98,24 \mathrm{~A}, \mathrm{a}$ \\
\hline & & & $89,92 \mathrm{~B}, \mathrm{a}$ & & $97,76 \mathrm{~A}, \mathrm{a}$ \\
\hline & & \multicolumn{4}{|c|}{ CV $2,82 \%$} \\
\hline \multirow{2}{*}{$3^{\circ}$ dia } & & \multirow{2}{*}{$\mathrm{P}$} & $1,56 \mathrm{~B}, \mathrm{a}$ & $\mathrm{R}$ & $1,92 \mathrm{~A}, \mathrm{a}$ \\
\hline & & & $0,36 \mathrm{~A}, \mathrm{~b}$ & & $1,26 \mathrm{~A}, \mathrm{~b}$ \\
\hline \multirow{2}{*}{$6^{\circ}$ dia } & & \multirow{2}{*}{$\mathrm{P}$} & $2,80 \mathrm{~B}, \mathrm{a}$ & $\mathrm{R}$ & $10,92 \mathrm{~A}, \mathrm{a}$ \\
\hline & & & $0,88 \mathrm{~A}, \mathrm{~b}$ & & $2,5 \mathrm{~A}, \mathrm{~b}$ \\
\hline \multirow{2}{*}{$9^{\circ}$ dia } & \multirow{4}{*}{ Esporulação (107conídios/mL) } & P & $15,04 \quad \mathrm{~B}, \mathrm{a}$ & $\mathrm{R}$ & $17,24 \mathrm{~A}, \mathrm{a}$ \\
\hline & & $P$ & $2,84 \mathrm{~A}, \mathrm{~b}$ & & $4,84 \mathrm{~A}, \mathrm{~b}$ \\
\hline \multirow{2}{*}{$12^{\circ}$ dia } & & $P$ & $17,24 \mathrm{~B}, \mathrm{a}$ & $\mathrm{R}$ & 21,64 A,a \\
\hline & & $P$ & $6,88 \mathrm{~A}, \mathrm{~b}$ & & $7,64 \mathrm{~A}, \mathrm{~b}$ \\
\hline
\end{tabular}

Médias seguidas de mesmas letras, maiúscula na coluna e minúscula na linha, não diferem estatisticamente entre si, pelo teste de Duncan $(\mathrm{P}=0,05)$. ${ }^{1}$ Fungo-padrão. ${ }^{2}$ Fungo - reisolado. 


\section{REFERÊNCIAS}

ALBUQUERQUE, A.C.; PEREIRA, K.C.A.; CUNHA, F.M., VEIGA, A.F.S.L.; ATHAYDE, A.C.R.; LUNAALVES LIMA, E.A. Patogenicidade de Metarhizium anisopliae var. anisopliae e Metarhizium anisopliae var. acridum sobre Nasutitermes coxipoensis (Holmgreen) (Isoptera: Temitidae). Neotropical Entomology, v.34, n.4, p.585-591, 2005.

ALMEIDA, J.C.; ALBUQUERQUE, A.C.; LUNA-ALVES LIMA, E.A. Viabilidade de Beauveria bassiana (Bals.) Vuill. reisolado de ovos, larvas e adultos de Anthonomus grandis (Boheman) (Coleoptera: Curculionidae) artificialmente infectado. Arquivos do Instituto Biológico, São Paulo, v.72, n.4, p.473-480, 2005.

ALVES, S.B. Fungos entomopatogenicos. In: (Ed.). Controle microbiano dos insetos. 2.ed. Piracicaba: FEALQ, 1998. p.289-370.

ALVES, S.B.; MORAIS, S.A. Quantificação de inóculo de patógenos de insetos. In: ALVES, S.B. (Ed.). Controle microbiano de insetos. 2.ed. Piracicaba: FEALQ, 1998. Cap. 23.

ALVES, S.B.; PEREIRA, R.M. Produção de fungos entomopatogênicos. In: ALVES, S.B. (Ed). Controle microbiano de insetos. 2ed. Piracicaba: FEALQ, 1998. Cap. 27.

ALVES, S.B.; ALMEIDA, J.E.M.; MOINO, A.; ALVES, L.F.A. Técnicas laboratorias. In: ALVES, S.B. (Ed.). Controle microbiano de insetos. 2.ed. Piracicaba: FEALQ, 1998. Cap. 20p.

CONSTANTINO, R. Chave ilustrada para identificação dos gêneros de cupins (Insecta: Isoptera) que ocorrem no Brasil. Papéis Avulsos de Zoologia, v.40, n.25, p.387-448, 1999.

FARIA, M.R. de; MAGALHÃES, B.P. O uso de fungos entomopatogênicos: situação e perspectivas. Biotecnologia Ciências \& Desenvolvimento, n.22, p.18-21, 2001.

FONTES, L.R.; MILANO, S. Termites as an urban problem in South America. Sociobiology, v.40, n.1, p.103-151, 2002.

GALLO, D.; NAKANO, O.; SILVEIRA NETO, S.; CARVALHO, R.P.L.; BAPTISTA, G.C.; BERTI FILHO, E.; PARRA, J.R.P.; ZUCCHI, A.R.; ALVES, S.B.; VENDRAMIM, J.D.; MARCHINI, L.C.; LOPES, J.R.S.; OMOTO, C. Entomologia agrícola. São Paulo: FEALQ, 2002. 920p.

GRACE, J.K. Biological control strategies for suppression of termites. Journal of Agricultural Entomology, v.14, n. 3, p. 281-289, 1997.

HANEL, H.A. A bioassay for measuring the virulence of the insect pathogenic fungus Metarhizium anisopliae (Mestch.) Sorokin (Fungi Imperfect) against the Nasutitermes exitiosus (Hill) (Isoptera, Termitidae). Zeitschrift ur Angewandte Entomologie, v.92, n.5 p.9-18, 1981.
LOUREIRO, E.S.; MONTEIRO, A.C. Seleção de isolados de Beauveria bassiana, Metarhizium anisopliae e Paecilomyces farinosus patogênicos para operárias de Atta sexdens sexdens (Linnaeus, 1758) (Hymenoptera: Formicidae). Arquivos do Instituto Biológico, São Paulo, v.71, n.1, p.35-40, 2004.

MEIKLE, W.G.; MERCADIER, G.; ROSENGAUS, R.B.; KIRK, A.A.; DEROUANÉ, F.; QUIMBY, P.C. Evaluation of an entomopathogenic fungus, Paecilomyces fumosoroseus (Wize) Brown \& Smith (Deuteromycota: Hyphomycetes) obtained from formosan subterranean termites (Isoptera: Rhinotermitidae). Journal of Applied Entomology, v.129, n.6, p.315-322, 2005.

MILNER, R.J. Application of biological control agents in mound building termites (Isoptera: Termitidae) experiences with Metarhizium anisopliae in Australia. Sociobiology, v.41, n.2, p.419-428, 2003.

MILNER, R.J.; STAPLES, J.A.; LUTTON, G.G. The effect of humidity on germination and infection of termites by Hyphomycetes, Metarhizium anisopliae. Journal of Invertebrate Pathology, v.69, n.1, p.64-69, 1997.

MILNER, R.J.; STAPLES, J.A.; LUTTON, G.G. The selection of an isolate of the Hyphomycetes fungus Metarhizium anisopliae, for control of termites in Australia. Biological Control, v.11, n.3, p.240-247, 1998.

RATH, A.C. The use of entomopathogenic fungi for control of termites. Biological Science and Technology, v.10, n.5, p.563-581, 2000.

SAMSON, R.A. Paecilomyces and some allied Hyphomycetes. Studies in Mycology, v.6, n.6, p.1-119, 1974.

SAMSON, R.A.; EVANS, H.C.; LATGÉ, J.P. Taxonomy of entomopathogenic. In: (Ed.) Atlas of Entomopathogenic Fungi. Berlin: Springer-Verlag, 1988.

SCORSETTI, A.C.; HUMBER, R.A.; DE GREGÓRIO, C.; LASTRA, C.C.L. New records of entomopathogenic fung infecting Bemisia tabaci and Trialeurodes vaporariorum pests of horticultural crops in Argentina. BioControl, v.53, n.5, p.787-796, 2008.

SILVA, V.C.A.; BARROS, R.; MARQUES, E.J. Suscetibilidade de Plutella xylostela (L.) (Lepidoptera: Plutellidae) aos fungos Beauveria bassiana (Bals.) Vuill. e Metarhizuim anisopliae (Mestsch.) Sorokin. Neotropical Entomology, v.32, n.4, p.653-658, 2003.

SPECHT, A.; AZEVEDO, J.L.; LUNA-ALVES LIMA, E.A.; BOLDO, J.T.; MARTINS, M.K.; LORINI, L.M.; BARRROS, N.M. Ocorrência do fungo entomopatogênico Isaria javanica (Frieder \& Bally) Samson \& HywellJones (Fungi, Sordariomycetes) em lagartas de Lonomia obliqua Walker (Lepidoptera, Saturniidae, Hemileucinae). Revista Brasileira de Entomologia, v.53, n.3, p.493-494, 2009. 
SUN, J.; FUXA, J.R.; HENDERSON, G. Sporulation of Metarhizium anisopliae and Beauveria bassiana on Coptotermes formosanus and in vitro. Journal of Invertebrate Pathology, v.81, n.2, p.78-85, 2002.

SUN, J.; FUXA, J.R.; HENDERSON, G. Effects of virulence, sporulation, and temperature on Metarhizium anisopliae and Beauveria bassiana laboratory transmission in Coptotermes formosanus. Journal of Invertebrate Pathology, v.84, n.1, p.38-46, 2003.

VILAS BOAS, A.M.; ANDRADE, R.M.; OLIVEIRA, J.V. Diversidade de cultura para a produção de fungos entomopatogênicos. Brazilian Archives of Biology and Technology, v. 39, n.1, p. 123-128, 1996.

WANG, C.; POWELL, J.E. Isolation and evaluation of Beauveria bassiana of control of Coptotermes formosanus and Reticulitermes flavipes (Isoptera: Rhinotermitiade). Sociobiology, v.41, n.2, p.248-255, 2003.

WRIGHT, M.S.; OSBRINK, W.L.A.; LAX, A.R. Transfer of entomopathogenic fungi among formosan subterranean termites and subsequent mortality. Journal of Applied Entomology, v.126, n.1, p.20-23, 2002.
WRIGHT, M.S.; CONNICK, W.J.; JACKSON, M.A. 2003. Use of Paecilomyces spp. as pathogenic agents against subterranean termites. U.S. Patent 20030095951.

WRIGHT, M.S.; RAINA, A.K.; LAX, A.R. A strain of the fungus Metarhizium anisopliae for controlling subterranean termites. Journal of Economic Entomology, v.98, n.5, p.1451$1458,2005$.

YANAGAWA, A.; YOKOHARI, F.; SHIMIZU, S. Defense mechanism of the termite, Coptotermes formosanus Shiraki, to entomopathogenic fungi. Journal of Invertebrate Pathology, v.97, n.2, p.165-170, 2008.

ZORZENON, F.J.; JUSTI JÚNIOR, J.; POTENZA, M.R.; CAMPOS, T.B. CANCELLO, E. M. Cupins: pragas em áreas urbanas. Boletim Técnico Instituto Biológico, n.18, p.4-66, 2006.

Recebido em 8/12/10

Aceito em 30/10/11 